rheuma plus $2016 \cdot 15: 96-99$

DOI 10.1007/s12688-015-0055-x

Online publiziert: 26. Januar 2016

(c) The Author(s) 2015. This article is available at

SpringerLink with Open Access.

CrossMark

Julia Held · Günter Weiss

Univ. Klinik für Innere Medizin VI, Medizinische Universität Innsbruck, Innsbruck, Österreich

\title{
Stellenwert der Anämie bei rheumatoider Arthritis
}

\section{Anämiedefinition und Einteilung der Anämieformen}

einer Prävalenz von $0,8 \%$ eine häufige Autoimmunerkrankung weltweit $[1,2]$. Neben der Gelenkbeteiligung in Form von persistierender Synovitis definiert sie sich auch über Autoantikörper, erhöhte Entzündungszeichen und systemische Organbeteiligung [3]. Diese systemische Komponente der RA spiegelt sich auch in den 2010 definierten Klassifikationskriterien des ACR (American College of Rheumatology) und der EULAR (European league against rheumatism) wider.

Neben der Anzahl der geschwollenen und druckschmerzhaften Gelenke und der Dauer der Symptome werden auch systemische Entzündungsparameter (BSG = Blutsenkungsgeschwindigkeit oder CRP = C-reaktives Protein) und Autoantikörper in Form von Rheumafaktorpositivität oder ACPA (, anti citrullinated protein antibody") miteinbezogen [4]. Die RA kann fast jedes Organsystem betreffen. Durch die der Krankheit zugrunde liegenden entzündlichen Prozesse kommt es bei RA-Patienten häufig zu einer Anämie, die als häufigste extraartikuläre Komplikation gesehen wird und mit einer starken Einschränkung der Lebensqualität einhergeht [5].

\section{Symptome der Anämie}

Die Symptome sind relativ unspezifisch und umfassen Fatigue (• Abb. 1), Schwindel, Schläfrigkeit und Schwäche. Außerdem kommt es zu einer reduzierten körperlichen Leistungsfähigkeit in Form von Belastungsdyspnoe und Tachykardie. Auch Kopfschmerzen und Konzentrationsschwäche werden berichtet $[6,7]$. Die Anämie wird von der WHO sogar zu einer der wichtigsten Faktoren für Morbidität und Mortalität gezählt.
Die am häufigsten verwendete Definition einer Anämie ist die Definition der WHO. Hier sind Frauen unter einem Hämoglobinwert von $12 \mathrm{~g} / \mathrm{dl}$ als anämisch zu betrachten, im Falle einer Schwangerschaft erst ab $11 \mathrm{~g} / \mathrm{dl}$. Bei Männern hingegen liegt der Grenzwert bei $13 \mathrm{~g} / \mathrm{dl}$ [8].

Die Einteilung der Anämieformen erfolgt meist nach der Morphologie und dem Hämoglobingehalt der Erythrozyten. Hier kann man mikrozytäre hypochrome, normozytäre normochrome oder makrozytäre hyperchrome Anämieformen unterscheiden. Repräsentativ für die erste Gruppe ist die Eisenmangelanämie, bei den normozytären normochromen Anämien finden sich die hämolytische Anämie, aplastische Anämien und die Anämie der chronischen Erkrankung. Hyperchrome makrozytäre Anämien sind häufig durch Folsäuremangel, oft im Rahmen einer Methotrexattherapie, Vitamin-B12Mangel, myelodysplastische Syndrome und medikamentös-toxische Ursachen begründet [9].

Bei Patienten mit rheumatoider Arthritis sind die zwei häufigsten Formen die Anämie der chronischen Erkrankung (Anemia of chronic disease, ACD) und die Eisenmangelanämie (Iron deficiency anemia, IDA) [10]. Die ACD wird durch die systemische Entzündung verursacht, meist handelt es sich um eine milde Anämie. Schwere Anämieformen mit einem Hämoglobinwert unter $10 \mathrm{~g} / \mathrm{dl}$ sind nur bei 3,4\% der Patienten zu finden und damit relativ selten [11]. Ursächlich für die Eisenmangelanämie sind meistens gastrointestinale Blutungen, die oft durch
Medikamente zur Therapie der RA, wie zum Beispiel NSAR (nichtsteroidale Antirheumatika), verursacht werden [12].

\section{Differenzierung der Anämie- formen}

Die Unterscheidung der zwei häufigsten Anämieformen ist oft nicht eindeutig, da ein wichtiger Parameter zur Differenzierung, wie zum Beispiel Serumferritin, das normalerweise mit dem Eisen in den Eisenspeichern korreliert, auch ein Akutphaseprotein ist und durch die krankheitsbedingte chronische Entzündung beeinflusst wird [13]. Zudem können auch Mischformen der ACD und IDA, wie auch jede andere Anämieform im Rahmen einer Anämie, entweder durch die Grunderkrankung oder durch krankheitsunabhängige Faktoren auftreten.

Die Anämie ist ein gemeinsames Symptom völlig unterschiedlicher Mechanismen; zurgezielten Therapie ist aber die genaue Differenzierung wichtig. Zur richtigen Interpretation der Laborparameter ist ein Einblick in die zugrunde liegenden Mechanismen hilfreich. Bei der ACD kommt es durch die entzündlich-rheumatische Grunderkrankung zu einer Aktivierung von Makrophagen und T-Zellen. Diese Zellen schütten dann ihrerseits proinflammatorische Zytokine, wie Interferon gamma, TNF alpha, Interleukin 1 und Interleukin 6, aus. Diese Zytokine wirken sich direkt negativ auf die Blutbildung aus. Einerseits hemmen sie die biologische Wirksamkeit von Erythropoetin, außerdem blockieren sie die Erythropoese und wirken zytotoxisch auf bereits gebildete Erythrozyten. Zudem kommt es zu einer Retention von Eisen in Zellen des retikuloendo- 


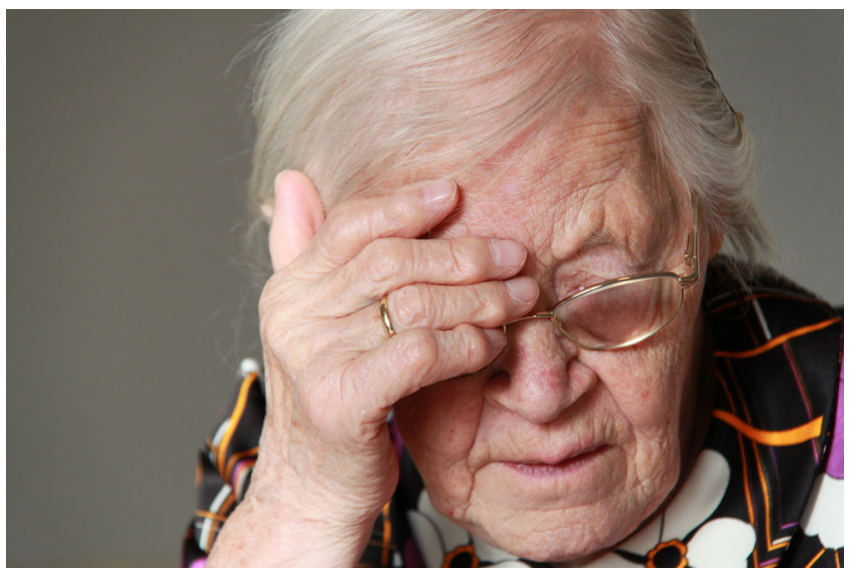

Abb. $1<$ Die Anämie geht mit einer starken Einschränkung der Lebensqualität einher. Symptome einer Anämie sind relativ unspezifisch und umfassen Fatigue, Schwindel, Schläfrigkeit und Schwäche (@) Sybille Leu/ panthermeda.net)

thelialen Systems (RES), wodurch die zirkulierenden Eisenspiegel sinken und die Verfügbarkeit dieses für die Hämoglobinsynthese essenziellen Metalls für erythroide Vorläuferzellen sinkt.

\section{》) Die Anämie ist ein gemeinsames Symptom unterschiedlicher Mechanismen}

Ein wichtiges Regulatormolekül des Eisenstoffwechsels ist Hepcidin. Seine Produktion in der Leber wird nicht nur durch Eisen, sondern auch durch proinflammatorische Zytokine (v. a. IL-6) stimuliert. Hepcidin bindet an das einzige zelluläre Eisenexportprotein, Ferroportin, und bewirkt dessen Degradation vor allem auf Makrophagen und Enterozyten, womit die Eisenrezirkulation aus Makrophagen und die Eisenaufnahme aus dem Darm blockiert werden. Daneben stimulieren die proinflammatorischen Zytokine die Eisenaufnahme und Speicherung in Makrophagen, was auch teilweise durch eine Reduktion der zirkulierenden Halbwertszeit von Erythrozyten bedingt ist. Typische Zeichen der ACD sind deshalb ein niedriger Eisenspiegel und eine erniedrigte Transferrinsättigung, die die Eisenbeladung des Transportproteins Transferrin widerspiegelt, sowie ein normaler oder erhöhter Ferritinwert.

Bei der Eisenmangelanämie hingegen kommt es aufgrund von übermäßigem Eisenverlust durch Blutungen, zum Beispiel bei NSAR-induzierte Enteropathien, oder aufgrund einer reduzierten Resorption zu einem Entleeren der Eisen- speicher. Diese leeren Eisenspeicher spiegeln sich in einem erniedrigten Ferritin sowie in niedrigen zirkulierenden Eisenspiegeln wider. Da die Erythrozyten aufgrund des niedrigen Eisens und der daraus resultierenden geringen Hämoglobinproduktion wenig Hämoglobin enthalten, ist diese Anämieform hypochrom und mikrozytär [14].

Die Relevanz der NSAR-induzierten Nebenwirkungen bei RA-Patienten ist nicht zu unterschätzen. In einer Studienpopulation mit einem Risikofaktor, eine gastrointestinale Nebenwirkung bei NSAR-Einnahme zu entwickeln, wurden bei $27 \%$ der Patienten unkomplizierte GI-Symptome beobachtet, 3\% wiesen sogar schwere GI-Komplikationen wie Blutungen oder Perforationen auf [15]. In einer anderen Kohorte wurden RA-Patienten, die regelmäßig NSAR einnahmen, untersucht. Falls sie eine Anämie oder eine okkulte gastrointestinale Blutung aufwiesen, wurde eine Kapselendoskopie durchgeführt. Hier konnte bei $68 \%$ der Patienten eine NSAR-induzierte Enteropathie festgestellt werden [16].

\section{Auswirkungen der Anämie auf die Aktivitätsbeurteilung der RA}

Die Aktivität der RA kann über verschiedenste Scores gemessen werden. Im klinischen Alltag wird häufig der DAS-28 ("disease activity score 28“) angewendet. Der DAS-28 berechnet die Aktivität der RA durch Schwellung und Druckschmerzhaftigkeit an 28 definierten Indikatorgelenken. Außerdem fließen systemische Entzündungsparameter, wie die Blutsenkungsgeschwindigkeit (BSG) oder das CRP, und die subjektive Krankheitsaktivitätseinschätzung des Patienten mittels VAS („visual analog scale“, von 0-100 mm) ein. Je nach Ergebnis kann bei einem DAS-28 unter 3,2 von einer niedrigen Krankheitsaktivität ausgegangen werden, ein Wert zwischen 3,2 und 5,1 spricht für eine mittlere Aktivität und ein Wert von über 5,1 für eine hohe Aktivität der Erkrankung.

Per se kann in Patienten mit einer höheren Krankheitsaktivität von einer vermehrten Anämieprävalenz ausgegangen werden, da sie aufgrund der erhöhten Entzündungsparameter und der häufigeren Einnahme von NSAR zwei der häufigsten Risikofaktoren aufweisen, um eine Anämie zu entwickeln. Jedoch konnte in Studien bewiesen werden, dass die Therapie der Anämie zu einer Reduktion der Krankheitsaktivität beiträgt. Eine kombinierte Therapie mit Eisen und Erythropoetin bewirkte eine signifikante Reduktion an geschwollenen Gelenken und auch eine deutliche Reduktion der VAS [17].

\section{Anämieauswirkung auf die Morbidität und Krankheits- progression}

Bei Patienten mit chronischen Erkrankungen ist die Lebensqualität ein wichtiger Faktor, der auch den Therapieerfolg mitbestimmt. Rheumapatienten mit Anämie zeigten im Vergleich zu nicht anämischen Rheumapatienten eine deutlich reduzierte Lebensqualität, was sich

Hier steht eine Anzeige.

Springer 
auch in der körperlichen Einschränkung im Rahmen der Grunderkrankung manifestiert [18]. Diese Beeinträchtigung kann durch den HAQ (Health Assessment Questionnaire) quantifiziert werden.

\section{) Rheumapatienten mit Anämie zeigten eine deutlich reduzierte Lebensqualität}

Anämische Patienten zeigten eine deutlich höhere körperliche Einschränkung als nicht anämische mit gleicher Krankheitsaktivität [19]. Dies spiegelt sich auch in verlängerten Krankenhausaufenthalten von RA-Patienten mit begleitender Anämie wider. Somit ist die Anämie nicht nur ein wichtiger Faktor für die Lebensqualität von RA-Patienten, sondern auch ein maßgeblicher Faktor für höhere Gesundheitskosten, verursacht durch die höhere Anzahl an Untersuchungen und den verlängerten stationären Aufenthalt [20].

Das Verhindern der Krankheitsprogression ist das vorrangige Ziel in der Therapie der RA. Bereits entstandene erosive Veränderungen am Knochen können auch durch moderne Therapiestrategien nicht mehr rückgängig gemacht werden. Somit ist es von besonderer Bedeutung, dass die Anämie ein unabhängiger Faktor für die radiologische Progression von $\mathrm{Ge}$ lenksschäden ist. Es zeigt sich eine klare Assoziation zwischen Ausmaß der Anämie und Progression der Veränderungen am Knochen [21].

\section{Entwicklung}

Im Vergleich zu früheren Daten ist die Prävalenz der Anämie bei der RA von 30-66 \% auf zirka $16 \%$ abgesunken [17, 22]. Die Ursache hierfür ist sicherlich die erhöhte Awareness für die Früherkennung der RA, die Therapie der Anämie, aber auch neue Therapieoptionen für die Behandlung der RA. Die ersten sogenannten Biologika sind seit 1999 für die Therapie der RA zugelassen. Seitdem kann, bei unzureichender Wirkung der cDMARDS (conventional diseasemodifying anti-rheumatic drugs), die Therapie um eines dieser Medikamen-

rheuma plus 2016 - 15:96-99 DOI 10.1007/s12688-015-0055-x

(c) The Author(s) 2015. This article is available at SpringerLink with Open Access.

\section{J. Held. G. Weiss \\ Stellenwert der Anämie bei rheumatoider Arthritis}

\section{Zusammenfassung}

Die Anämie ist eine häufige Komplikation der rheumatoiden Arthritis (RA). Die Anämie hat negative Auswirkungen auf den Verlauf der RA und die Lebensqualität der Patienten. Neben entzündungsabhängigen Veränderungen in der Eisenhomöostase und einer eingeschränkten biologischen Aktivität von Erythropoetin spielen auch Vitaminmangel, chronischer Blutverlust und Nebenwirkungen von RA-Therapeutika eine wichtige Rolle für die Anämieentstehung. Aufgrund der morbiditätsfördernden Effekte der Anämie sollte diese als eine abklärungs- und therapiebedürftige Komplikation bei RA wahrgenommen werden. Trotz einer deutlichen Abnahme der Prävalenz der Anämie in den vergangenen Jahren durch moderne Therapiestrategien findet sich diese bei RA-Patienten immer noch doppelt so häufig wie in der altersgematchten Normalpopulation.

Schlüsselwörter

Anämie · Rheumatoide Arthritis · Eisenmetabolismus - Erythropoetin · Zytokine

\section{Significance of anemia in rheumatoid arthritis}

\section{Abstract}

Anemia is a frequently observed comorbidity in patients with rheumatoid arthritis (RA). It negatively impacts on disease activity and physical performance and accelerates disease progression. Besides inflammation-induced changes in iron homeostasis, impaired erythroid cell proliferation and erythropoietin activity, vitamin deficiencies, blood loss, and side effects of RA treatments contribute to the development of anemia. Because anemia is associated with an increased morbidity of RA patients, awareness should be attributed to the clarification of the cause of anemia resulting in appropriate treatment. Although modern therapeutic strategies of RA lead to reduction in anemia prevalence in RA patients, it is still a relevant clinical problem, and is found more than twice as frequently in RA patients than in age-matched controls.

\section{Keywords}

Anemia $\cdot$ Rheumatoid Arthritis · Iron metabolism · Erythropoietin · Cytokines te erweitert werden. Daraus ergeben sich eine deutlich bessere Krankheitsaktivitätskontrolle und ein reduzierter Gebrauch an NSAR, die wiederum in einer Reduktion der Anämie resultieren.

\section{Therapie der Anämie}

Aufgrund der gezeigten unterschiedlichen Mechanismen, die der Anämieentwicklung zugrunde liegen, ist die effektivste Therapie die Behandlung der auslösenden Ursache. Entscheidend ist es auch, die genauen Ursachen der Anämie zu kennen und eine IDA von einer ACD oder einer ACD + IDA zu unterscheiden. Eine Eisensubstitution ist im Rahmen der Eisenmangelanämie oder einer ACD + IDA die Therapie der Wahl, jedoch sollte hierbei immer auch die Ursache des Eisenmangels abgeklärt werden. Bei einer
ACD kann eine Eisentherapie mitunter wegen der inflammationsbedingten Eisenretention nicht effektiv sein, weshalb in diesem Fall auch rekombinante Erythropoetinpräparate zum Einsatz kommen können. Abzuklären sind auch andere Ursachen, wie Vitaminmangelsyndrome, die dann entsprechend zu behandeln sind. Bluttransfusionen sind nur als Notfalltherapie bei Patienten mit schweren, lebensbedrohlichen Anämien indiziert [23].

\section{Fazit}

Die Anämie ist eine häufige Komplikation bei rheumatoider Arthritis, der unterschiedlichste Mechanismen zugrunde liegen. Die Anämie hat negative Auswirkungen auf den Verlauf der RA und die Lebensqualität der Patienten. 
Die Anämie sollte auch als eine abklärungsbedürftige und therapiebedürftige Komplikation bei RA wahrgenommen werden. Trotz einer deutlichen Abnahme der Prävalenz der Anämie in den vergangenen Jahren, findet sich diese bei RA-Patienten immer noch doppelt so häufig wie in einer altersgematchten Normalpopulation [24].

\section{Korrespondenzadresse}

\section{Dr. J. Held}

Univ. Klinik für Innere Medizin VI, Medizinische Universität Innsbruck

Anichstraße 35, 6020 Innsbruck, Österreich julia.held@tirol-kliniken.at

\section{Einhaltung ethischer Richtlinien}

Interessenkonflikt. J. Held und G. Weiss geben an, dass kein Interessenkonflikt besteht.

Dieser Beitrag beinhaltet keine Studien an Menschen oderTieren.

Open Access. This article is distributed under the terms of the Creative Commons Attribution 4.0 International License (http://creativecommons.org/ licenses/by/4.0/), which permits unrestricted use, distribution, and reproduction in any medium, provided you give appropriate credit to the original author(s) and the source, provide a link to the Creative Commons license, and indicate if changes were made.

\section{Literatur}

1. Klarenbeek NB et al (2010) Recent advances in the management of rheumatoid arthritis. BMJ 341:c6942 doi:10.1136/bmj.c6942

2. O'Dell JR (2004) Therapeutic strategies for rheumatoid arthritis. N Engl J Med 350(25):2591-2602

3. Scott DL, Wolfe F, Huizinga TW (2010) Rheumatoid arthritis. Lancet 376(9746):1094-1108. doi:10.1016/S0140-6736(10)60826-4

4. Aletaha D et al (2010) Rheumatoid arthritis classification criteria: an american college of rheumatology/european league against rheumatism collaborative initiative. Ann Rheum Dis 69:1580-1588. doi:10.1136/ard.2010.138461

5. Wahle M (2012) Anämie bei Patienten mit rheumatoider Arthritis. ZRheumatol 71(10):864-868

6. McLean E et al (2009) Worldwide prevalence of anaemia, WHO vitamin and mineral nutrition information system, 1993-2005. Public Health Nutr 12(4):444-454

7. Behrends J, Bischofberger J, Deutzmann R (2012) Duale Reihe: Physiologie Georg Thieme, Stuttgart

8. Beutler E, Waalen J (2006) The definition of anemia: what is the lower limit of normal of the blood hemoglobin concentration? Blood 107(5):1747-1750. doi:10.1182/blood-2005-073046

9. Herold G (2014) Innere Medizin. Gerd Herold, Köln

10. Peeters HRM et al (1996) Course and characteristics of anaemia in patients with rheumatoid arthritis of recent onset. Ann Rheum Dis 55(3):162-168

11. Wolfe F, Michaud K (2006) Anemia and renal function in patients with rheumatoid arthritis. $J$ Rheumatol 33(8):1516-1522

12. Francis J et al (2005) Iron deficiency anaemia in chronic inflammatory rheumatic diseases: low mean cell haemoglobin is a better marker than low mean cell volume. Ann Rheum Dis 64:787-788

13. Ravindran V, Sandeep J, Mathur DS (2008) The differentiation of anaemia in rheumatoid arthritis: parameters of iron-defciency in an Indian rheumatoid arthritis population. Rheumatol Int 28(6):507-511

14. Weiss G, Goodnough LT (2005) Anemia of chronic disease. NEngl J Med 352:1011-1023
15. Lanas A, Boers M, Nuevo J (2015) Gastrointestinal events in at-risk patients starting non-steroidal anti-inflammatory drugs (NSAIDs) for rheumatic diseases: the EVIDENCE study of European routine practice. Ann Rheum Dis 74(4):675-681

16. Tachecí I, Bradna P, Douda $T$, Baštecká $D$, Kopáčová M, Rejchrt S, Bureš J (2013) NSalDinduced Enteropathy in rheumatoid arthritis patients with chronic occult gastrointestinal bleeding: a prospective capsule endoscopy study. Gastroenterol Res Pract 2013:268382 doi:10.1155/2013/268382

17. Wilson A et al (2004) Prevalence and outcomes of anemia in rheumatoid arthritis: a systematic review of the literature. Am J Med 116(Suppl 7A):50S-57S

18. Kaltwasser JP, Kessler U, Gottschalk R (2001) Effect of recombinant human erythropoietin and intravenous iron on anemia and disease activity in rheumatoid arthritis. J Rheumatol 28(11):2430-2436

19. Han C et al (2007) Association of anemia and physical disability among patients with rheumatoid arthritis. JRheumatol 34(11):2177-2182

20. Zlateva $\mathrm{G}$ et al (2010) Burden of anemia in patients with osteoarthritis and rheumatoid arthritis in French secondary care. BMC Geriatr 10:59 doi:10.1186/1471-2318-10-59

21. Möller B etal (2014) Anaemia may add information to standardised disease activity assessment to predict radiographic damage in rheumatoid arthritis: a prospective cohort study. Ann Rheum Dis 73:691-696. doi:10.1136/annrheumdis-2012202709

22. Furst DE et al (2009) Prevalence of low hemoglobin levels and associations with other disease parameters in rheumatoid arthritis patients: evidence from the CORRONA registry. Clin Exp Rheumatol 27(4):560-566

23. Weiss G, Schett G (2013) Anemia in inflammatory rheumatic disease. Nat Rev Rheumatol 9(4):205-215

24. Eisele L (2013) Prevalence and incidence of anemia in the german Heinz Nixdorf recall study. Ann Hematol 92(6):731-737

Hier steht eine Anzeige. 